\title{
Diagnostic accuracy of demarcation using magnifying endoscopy with narrow-band imaging for Helicobacter pylori-uninfected undifferentiated-type early gastric cancer
}

\author{
Yusuke Horiuchi $^{1}{ }^{1}$. Junko Fujisaki ${ }^{1}$. Noriko Yamamoto ${ }^{2}$. Shoichi Yoshimizu ${ }^{1}$. Akiyoshi Ishiyama ${ }^{1}$. \\ Toshiyuki Yoshio $^{1} \cdot$ Toshiaki Hirasawa $^{1}$ - Yorimasa Yamamoto ${ }^{3} \cdot$ Masatsugu Nagahama $^{3} \cdot$ Hiroshi Takahashi $^{3}$. \\ Tomohiro Tsuchida ${ }^{1}$
}

Received: 11 January 2018 / Accepted: 9 March 2018 / Published online: 13 March 2018

(c) The International Gastric Cancer Association and The Japanese Gastric Cancer Association 2018

\begin{abstract}
Background It is unknown how the accuracy rate of diagnostic demarcation of undifferentiated-type early gastric cancers (UD-type EGCs) differs between patients uninfected with Helicobacter pylori (H. pylori) and patients with and without $H$. pylori eradication. This study assessed and compared the diagnostic accuracy rate in the $H$. pylori-uninfected group and the non- $H$. pylori eradication and $H$. pylori eradication groups.

Methods Subjects were 81 patients with 81 lesions who underwent endoscopic submucosal dissection between January 2010 and January 2015. There were 21 lesions in the H. pylori-uninfected group, 27 in the H. pylori eradication group, and 33 in the non- $H$. pylori eradication group. The rate in the $H$. pylori-uninfected group was separately compared with the rates in the non- $H$. pylori eradication and $H$. pylori eradication groups, as was inflammatory cell infiltration.

Results The diagnostic accuracy rates were $60.6 \%$ in the non- $H$. pylori eradication group, $92.2 \%$ in the $H$. pylori eradication group, and $100 \%$ in the H. pylori-uninfected group; the rate was significantly higher in the H. pylori-uninfected group than in the non- $H$. pylori eradication group, but no significant difference was observed between the H. pylori-uninfected group and the $H$. pylori eradication group. In addition, the $H$. pylori-uninfected and $H$. pylori eradication groups showed no significant differences in neutrophil infiltration and intestinal metaplasia, whereas the state in the $H$. pylori-uninfected group was significantly milder.

Conclusions Because neutrophil infiltration was classified as mild or none, the accuracy of diagnostic demarcation was high in the H. pylori-uninfected and H. pylori eradication groups.
\end{abstract}

Keywords Gastric cancer · Endoscopic resection · Helicobacter pylori - Endoscopic gastrointestinal surgery

\section{Introduction}

Since the indications for endoscopic submucosal dissection (ESD) were expanded to include undifferentiated-type early gastric cancers (UD-type EGCs) [1-3], improvements

Yusuke Horiuchi

yusuke.horiuchi@jfcr.or.jp

1 Department of Gastroenterology, Cancer Institute Hospital, 3-10-6 Ariake, Koto-ku, Tokyo 135-8550, Japan

2 Department of Pathology, Cancer Institute Hospital, Tokyo, Japan

3 Department of Gastroenterology, Showa University Fujigaoka Hospital, Yokohama, Japan in preoperative diagnostic ability have been a concern. Diagnosis of demarcation of UD-type EGCs is known to be difficult because of the presence of proliferative zone extensions [4]. We previously demonstrated the utility of magnifying endoscopy with narrow-band imaging (ME-NBI) for diagnosis of demarcation in both ESD and surgical cases. When the diagnostic accuracy of demarcation was assessed, the rate of diagnostic accuracy with ME-NBI was approximately $80 \%$, indicating that ME-NBI has an add-on effect of approximately $30 \%$ compared with conventional white light imaging (WLI). ME-NBI was suggested to be useful in diagnosis of UD-type EGCs [5, 6]. Inflammatory cell infiltration appeared to be a cause of misdiagnosis, suggesting that resolution of inflammation could contribute to accurate diagnosis of demarcation. 
Helicobacter pylori (H. pylori) is a major cause of inflammation. We assumed that the resolution of inflammation following eradication of the infection might improve the diagnostic accuracy of demarcation. Therefore, we assessed the rates of diagnostic accuracy of demarcation with ME-NBI in patients with UD-type EGCs who underwent $H$. pylori eradication and patients who did not. We found that although the rate among those without $H$. pylori eradication was approximately $60 \%$, the rate in those with $H$. pylori eradication was much higher, at $90 \%$ or greater [7].

$H$. pylori tests are positive in the majority of patients with gastric cancer $[8,9]$. However, $H$. pylori-uninfected early gastric cancer has also been reported, with a low prevalence of $0.66 \%$ [10]. In addition, undifferentiated type is reported to be predominant in $H$. pylori-uninfected early gastric cancer [10]. The presence of $H$. pylori-uninfected UD-type EGCs was confirmed at our hospital with a low frequency (about 20\% of all UD-type EGCs) among patients who underwent ESD [11].
It is expected that the relative prevalence of $\mathrm{H}$. pyloriuninfected UD-type EGCs will increase in the future, as the rate of $H$. pylori infection declines.

The endoscopic and pathological features of $\mathrm{H}$. pyloriuninfected UD-type EGCs are shown in Fig. 1. We previously reported that compared with $H$. pylori-positive UDtype EGCs, $H$. pylori-uninfected UD-type EGCs are more likely to be intramucosal carcinoma with a smaller diameter, more frequently confined to the proliferative zone of the mucosa, and that many cases are macroscopically flat, located in the lower region of the stomach, and histologically signet ring cell carcinoma [11, 12]. In our previous study, we did not assess the rate of diagnostic accuracy of demarcation of $H$. pylori-uninfected UD-type EGCs, and there have been no reports regarding the rate from any other institutions.

In the present study, we compared three groups of patients with UD-type EGCs to determine the rate of diagnostic accuracy of demarcation in the $H$. pylori-uninfected group

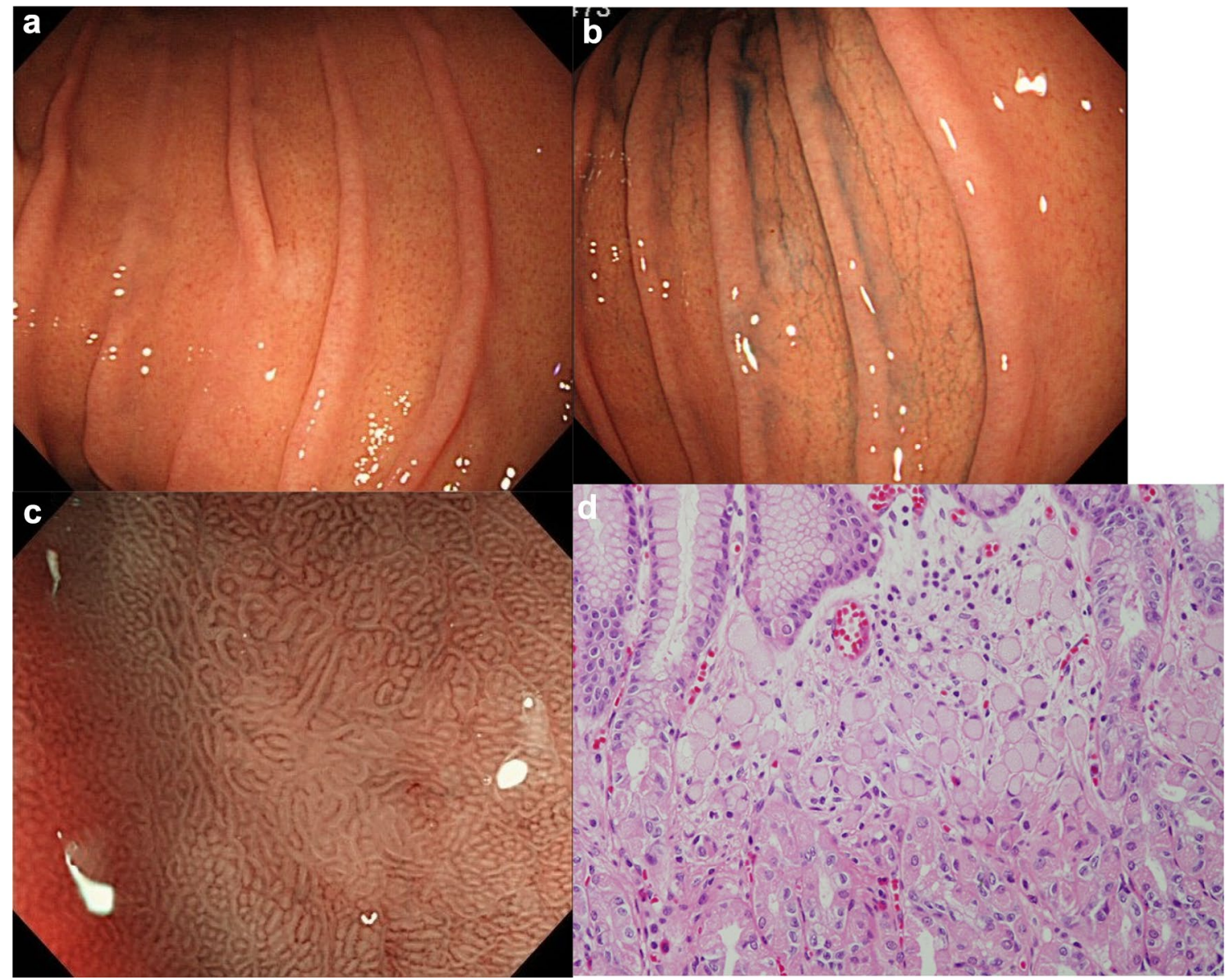

Fig. 1 Endoscopic and pathologic findings of Helicobacter pyloriuninfected undifferentiated-type cancers. a Endoscopic finding with white light imaging. A discolored area is relatively clearly recognizable. b Chromoendoscopic finding. c Finding of magnifying endos- copy with narrow-band imaging. An extended intervening part is recognizable. d Pathologic finding after endoscopic therapy. Signet ring cell carcinoma is confined to the proliferative zone of the mucosa 
and to reveal how it would differ from the rates in the non- $H$. pylori eradication and $H$. pylori eradication groups.

\section{Patients and methods}

This study was performed retrospectively at a single center. The subjects were 81 patients with 81 lesions that were detected on routine examination as $\leq 20$-mm lesions without metastasis or ulceration, diagnosed as UD-type EGC on biopsy, and treated with ESD between January 2010 and February 2015. Subjects were consecutive patients examined by two endoscopists certified by the Japan Gastroenterological Endoscopy Society. All endoscopic images and histopathological specimens could be re-examined. We included cases in which postoperative histopathological specimens were UD-type EGC and excluded cases in which postoperative histopathological specimens were differentiated-type cancer.

Patients in the eradication group met these inclusion criteria: confirmation during the first examination of a negative H. pylori antibody test (E-plate Eiken ${ }^{\circledR}$, Eiken Chemical Co., Ltd., Tokyo, Japan) or a negative ${ }^{13} \mathrm{C}$ urea breath test (UBIT, Otsuka Pharmaceutical Co., Ltd., Tokushima, Japan) in patients who had undergone $H$. pylori eradication at our or another hospital; and confirmation of a negative urea breath test performed at least 4 weeks after the start of H. pylori eradication in patients who were positive for $H$. pylori antibodies or had a positive urea breath test during the first examination at our hospital.

Moreover, the state of being uninfected with $H$. pylori should be determined by several different types of tests to exclude false negative results on each test [10].

H. pylori-uninfected status was defined by the following six criteria: (1) no prior $H$. pylori eradication, (2) urea breath test negative, (3) H. pylori antibody (E-plate Eiken ${ }^{\circledR}$, Eiken Chemical Co., Ltd.) negative, (4) pepsinogen test negative (cutoff level of positive: pepsinogen [PG] I $\leq 70 \mathrm{ng} / \mathrm{mL}$ and PG I/II ratio $\leq 3)$, (5) endoscopically confirmed positive regular arrangement of collecting venules in the lower gastric body [13], and (6) histologically confirmed H. pyloriuninfected status and inflammatory cell infiltration activity negative by the updated Sydney system [14].

Gastric cancers that did not meet at least one of the above six criteria and conditions of eradication were defined as $H$. pylori non-eradication.

In all patients, preoperative endoscopic and histopathological images were reviewed by three endoscopists with 10 years or more of experience in magnifying endoscopy and certified by the Japan Gastroenterological Endoscopy Society. First, using magnifying endoscopy (GIF-H260Z: Olympus Medical Systems, Tokyo, Japan), indigo carmine endoscopy with WLI was performed to demarcate lesions, which were photographed. Diagnoses were then made in reference to the following findings, on which we previously reported in relation to their usefulness for diagnosis of demarcation of UD-type EGCs: expansion of pathological intercrypt distance (extended intervening part) (Fig. 2), wavy microvessels, and corkscrew pattern on ME-NBI images [5, 16]. WLI was first performed to demarcate the lesions, followed by ME-NBI. A demarcation line is defined as existence of extended intervening parts, wavy microvessels, and corkscrew pattern between a mucosal lesion and background mucosa. The demarcation lines, determined as the utmost oral and utmost anal sites of the lesion, were marked with argon plasma coagulation (APC) (Fig. 3). The ME-NBI findings were compared with the results of postoperative pathological examination. A diagnosis was considered accurate when both the utmost oral and utmost anal sites marked in ME-NBI were consistent with the results of postoperative pathological examination (Fig. 4), and the rates of diagnostic accuracy were calculated. Cases in which only either the utmost oral or utmost anal site of the lesion was consistent were regarded as inconsistent. Given that the resected
Fig. 2 Intercrypt distance expansion. Compared with the noncancerous region, the cancerous region shows expansion of the distance between glands (intercrypt distance)

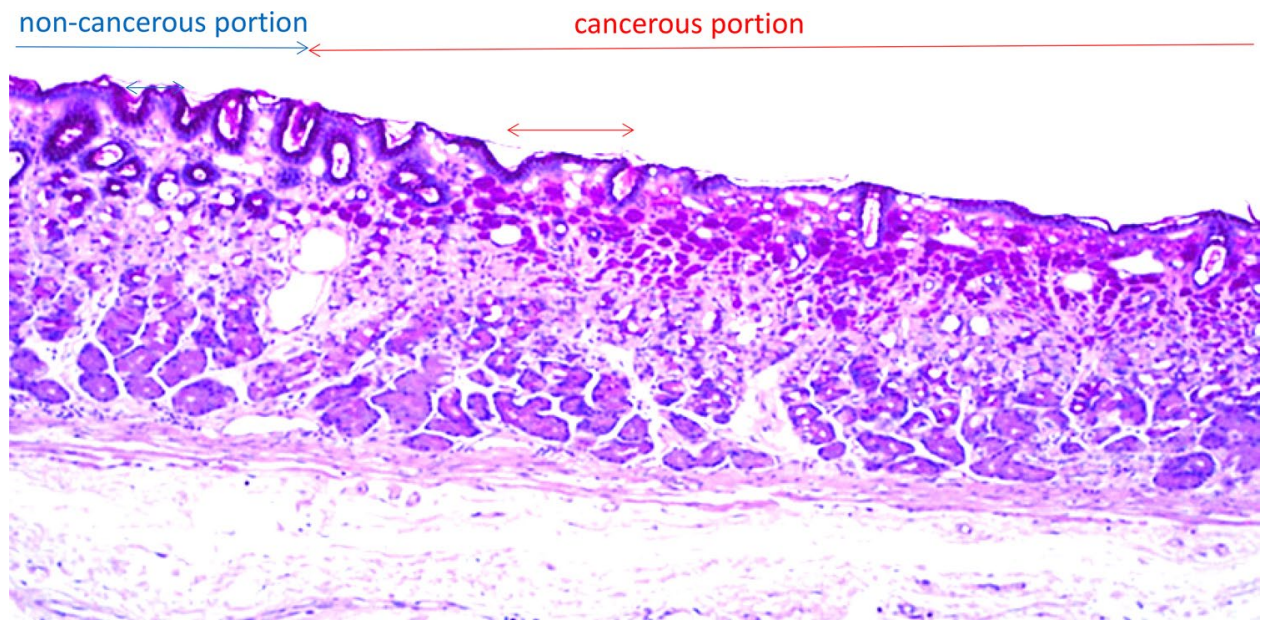


Fig. 3 Results of argon plasma coagulation marking. a Whole lesion depicted by white light imaging. b Whole lesion depicted by narrow-band imaging (NBI without magnification). Blue arrow indicates oral margin, and yellow arrow indicates anal margin. c Margin on utmost anal side depicted by NBI with high magnification. d Margin on utmost oral side depicted by NBI with high magnification. e Demarcation line marked with argon plasma coagulation (APC). $\mathbf{f}$ Image of whole lesion after circumferential marking prior to endoscopic submucosal dissection

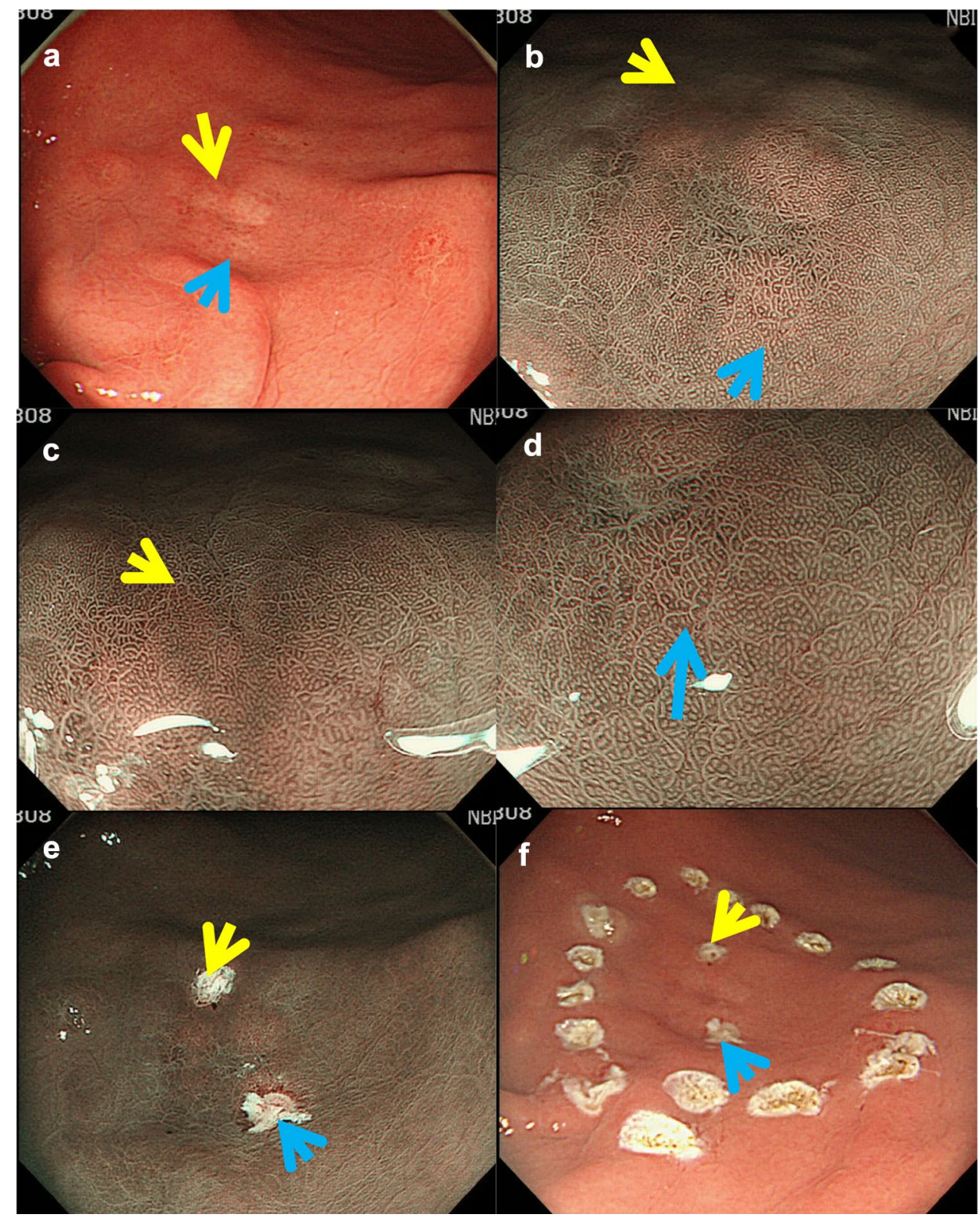

specimens had been cut into 2-mm slices, the acceptable error for accurate diagnosis was set at $1 \mathrm{~mm}$ or less.

Each patient was also examined for proliferative zone extension distance, intercrypt distance, and inflammatory cell infiltration. We defined the distance of cancer that develops along the proliferative zone as "proliferative zone extension distance". Proliferative zone extension and intercrypt distances were measured by light microscopy at 100 times magnification. Proliferative zone extension distance was defined as the distance between APC markings. Intercrypt distance was measured at 5-10 points each in the cancerous and noncancerous regions, and mean distances were calculated from the measurements. The mean distance ratio of the noncancerous region to the cancerous region (mean intercrypt distance ratio) was calculated. The intercrypt distance was defined as the distance between the center of a crypt and the center of an adjacent crypt. As for inflammatory cell infiltration, histological grades of monocyte infiltration, neutrophil infiltration, atrophy, and intestinal metaplasia were divided into two groups, normal-to-mild and moderate-to-marked, according to the updated Sydney classification [14].

In addition, the following data were collected and separately compared between the $H$. pylori-uninfected and non$H$. pylori eradication groups and between the $H$. pyloriuninfected and $H$. pylori eradication groups: age, sex, site of lesion, color of lesion, macroscopic type, depth of tumor invasion, histological type, tumor diameter, curability, adverse events, endoscopic findings of the APC-marked site, and presence or absence of atrophy around the lesion. 


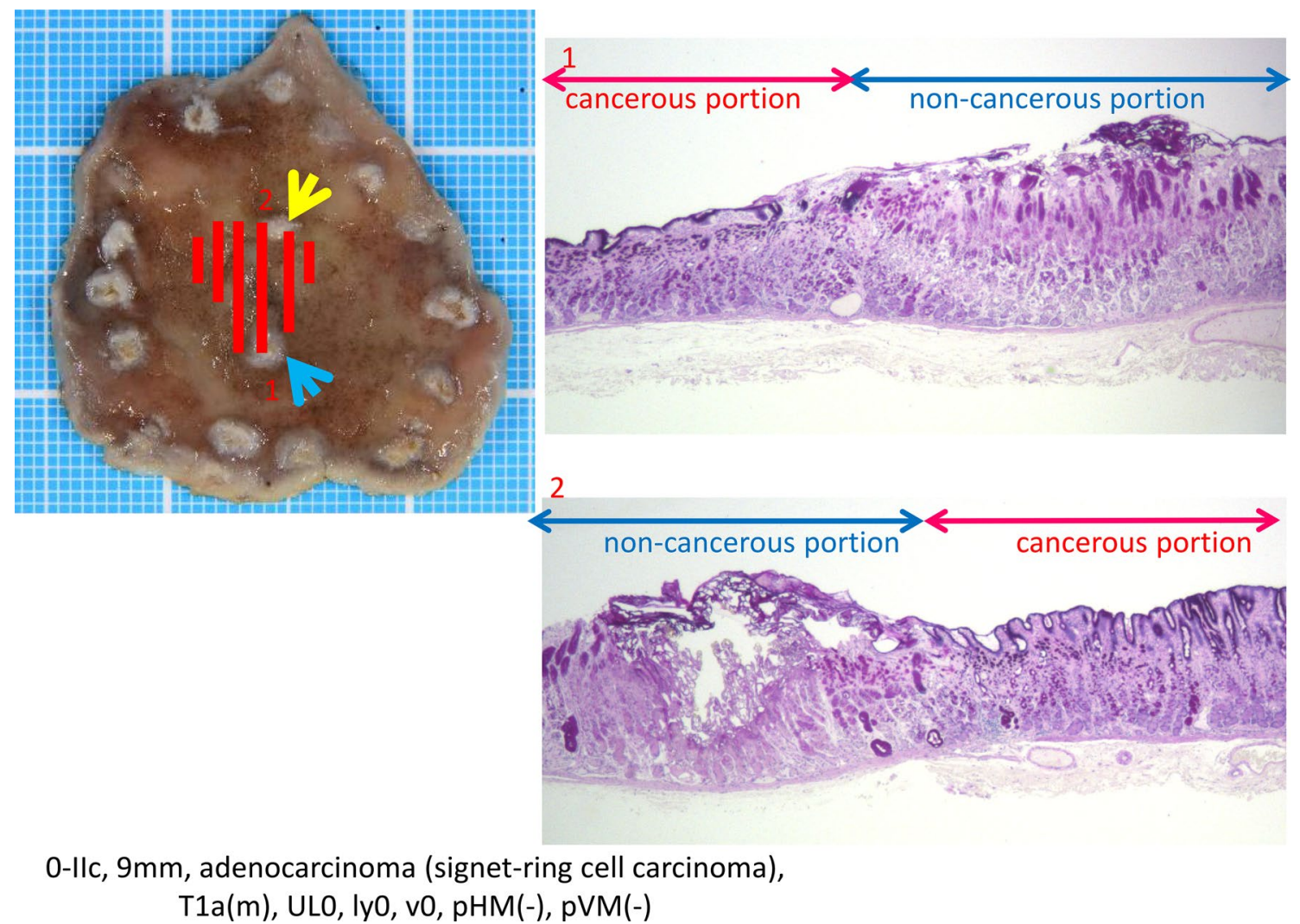

Fig. 4 Histopathological specimen after endoscopic submucosal dissection in case shown in Fig. 3. Margin between cancerous and noncancerous portions marked with argon plasma coagulation (APC) was consistent with that determined by histopathological examination.

The study was approved by the institutional review board at the Cancer Institute Hospital (IRB No. 2015-1009).

\section{Statistical analysis}

Fisher's exact test was performed for comparison between the two groups. For age, tumor diameter, proliferative zone extension distance, mean intercrypt distance ratio, mean values, and standard deviations were calculated and analyzed using the $t$ test and $F$ test. We performed the Mann-Whitney $U$ test to compare the eradication and non-eradication groups, because the specimens were not matched but independent.

Under the guidance of statistical experts, we determined the significance level as follows. Because we were interested in two pairwise comparisons (H. pylori-uninfected group vs. non- $H$. pylori eradication group, and $H$. pylori-uninfected group vs. H. pylori eradication group) with the $H$. pyloriuninfected group as the control group, we set the significance level to $<0.05 / 2$ using Bonferroni's method.

StatView software version 5.0 (SAS ${ }^{\circledR}$ Institute Inc., Cary, $\mathrm{NC}$, USA) was used for analysis.
Yellow and blue arrows indicate APC markings, and red lines (1 and 2 ) correspond to margins determined by histopathological examination

\section{Results}

There were 27 patients who had undergone $H$. pylori eradication, confirmed by urea breath tests at our hospital, along with 33 patients who had not undergone $H$. pylori eradication, and 21 patients who were $H$. pylori uninfected. The subjects included in this study were divided into $H$. pylori-uninfected, non- $H$. pylori eradication, and $H$. pylori eradication groups (Table 1). The H. pylori-uninfected and non- $H$. pylori eradication groups were then compared (in the following section, the data for the $H$. pylori-uninfected group are presented first, followed by those for the non- $H$. pylori eradication group). Macroscopically, the flat type was observed in 9 of the 21 lesions (42.9\%) and 1 of the 33 lesions (3.0\%). More flat-type lesions were found in the H. pylori-uninfected group. The mean tumor diameter in the two groups was $7.3 \pm 5.2 \mathrm{~mm}$ and $14.6 \pm 9.0 \mathrm{~mm}$, respectively, and significantly smaller in the $H$. pylori-uninfected group. Intramucosal carcinoma was observed in all 21 lesions (100\%) and 28 of the 33 lesions $(84.8 \%)$, while signet ring cell carcinoma was observed in all 21 lesions (100\%) and 23 of the 33 lesions (69.7\%). The proportions of both types of carcinoma were significantly 
Table 1 Comparison of accuracy in non-eradication and uninfected cases

\begin{tabular}{|c|c|c|c|c|c|}
\hline$n=81$ & Non-eradication $n=33$ & Eradication $n=27$ & Uninfected $n=21$ & $\begin{array}{l}P \text { value } \\
\text { Non-erad- } \\
\text { ication vs. } \\
\text { uninfected }\end{array}$ & $\begin{array}{l}P \text { value } \\
\text { Eradication } \\
\text { vs. unin- } \\
\text { fected }\end{array}$ \\
\hline Age & $55.7 \pm 12.3$ & $56.0 \pm 10.8$ & $52.5 \pm 9.3$ & N.S. & N.S. \\
\hline Male & $19(57.6)$ & $16(59.3)$ & $11(52.4)$ & N.S. & N.S. \\
\hline Color (discolored:reddish) & $30(91.0): 3(9.0)$ & $23(85.2): 4(14.8)$ & $21(100): 0$ & N.S. & N.S. \\
\hline \multicolumn{6}{|l|}{ Tumor-occupied site } \\
\hline Upper region & $3(0.9)$ & $3(11.1)$ & $0(0)$ & N.S. & N.S. \\
\hline Middle region & $13(39.4)$ & $14(51.9)$ & $8(38.1)$ & & \\
\hline Lower region & $17(51.5)$ & $10(37.0)$ & $13(61.9)$ & & \\
\hline \multicolumn{6}{|l|}{ Macroscopic types } \\
\hline Flat/depressed & $1(3.0): 32(97.0)$ & $3(11.1) / 24(88.9)$ & $9(42.9) / 12(57.1)$ & 0.0004 & 0.0184 \\
\hline \multicolumn{6}{|l|}{ Depth of tumor invasion } \\
\hline Intramucosal & $28(84.8)$ & $23(85.2)$ & $21(100)$ & N.S. & N.S. \\
\hline Submucosal (SM) & $5(15.2)$ & $4(14.8)$ & $0(0)$ & N.S. & N.S. \\
\hline $\mathrm{SM}<500 \mu \mathrm{m}$ & $3(9.0)$ & $3(11.1)$ & 0 & N.S. & N.S. \\
\hline $\mathrm{SM} \geq 500 \mu \mathrm{m}$ & $2(6.2)$ & $1(3.7)$ & 0 & N.S. & N.S. \\
\hline \multicolumn{6}{|l|}{ Histological type } \\
\hline Signet ring cell carcinoma & $23(69.7)$ & $17(63.0)$ & $21(100)$ & 0.0043 & 0.0064 \\
\hline Poorly differentiated & $10(30.3)$ & $10(37.0)$ & 0 & 0.0043 & 0.0064 \\
\hline \multicolumn{6}{|l|}{ Adverse event } \\
\hline Hemorrhage & $1(3.7)$ & $1(3.7)$ & $0(0)$ & N.S. & N.S. \\
\hline Perforation & 0 & 0 & 0 & N.S. & N.S. \\
\hline \multicolumn{6}{|l|}{ Curability } \\
\hline Complete $(\mathrm{R} 0)$ resection & $32(97.0)$ & $27(100)$ & $21(100)$ & N.S. & N.S. \\
\hline Incomplete resection (horizontal margin + ) & $1(3.0)$ & $0(0)$ & $0(0)$ & N.S. & N.S. \\
\hline Mean diameter of tumor $(\mathrm{mm})$ & $14.6 \pm 9.0$ & $13.6 \pm 7.3$ & $7.3 \pm 5.2$ & 0.0002 & 0.0008 \\
\hline $\begin{array}{l}\text { Mean intercrypt distance ratio }(95 \% \mathrm{CI}) \\
\text { (cancerous region/noncancerous region) }\end{array}$ & $1.59(1.40-1.78)$ & $1.94(1.69-2.20)$ & $1.96(1.70-2.21)$ & 0.0103 & N.S. \\
\hline Accuracy & $20 / 33(60.6)$ & $25 / 27(92.6)$ & $21 / 21(100)$ & 0.0007 & N.S. \\
\hline Proliferative zone extension distance $(\mu \mathrm{m})$ & $310.3 \pm 487.6$ & $362.3 \pm 494.5$ & $230.4 \pm 380.4$ & N.S. & N.S. \\
\hline Atrophy around lesion & $21(63.6)$ & $12(44.4)$ & 0 & $<0.0001$ & 0.0004 \\
\hline \multicolumn{6}{|c|}{ ME-NBI of the marked sites (utmost oral and utmost anal sites) } \\
\hline Extended intervening part & $42 / 66(63.6)$ & $38 / 54(70.4)$ & $41 / 42(97.6)$ & $<0.0001$ & 0.0003 \\
\hline Wavy-micro vessels & $17 / 66(25.8)$ & $12 / 54(22.2)$ & $1 / 42(2.4)$ & 0.0011 & 0.0055 \\
\hline Corkscrew pattern & $7 / 66(10.6)$ & 4/54 (7.4) & 0 & N.S. & N.S. \\
\hline \multicolumn{6}{|c|}{ Inflammation of the background mucosa-normal-mild ratio } \\
\hline Atrophy & $9 / 33(27.3)$ & $12 / 27(44.4)$ & $21 / 21(100)$ & $<0.0001$ & $<0.0001$ \\
\hline Intestinal metaplasia & $22 / 33(66.7)$ & $21 / 27(77.8)$ & 21/21 (100) & 0.0038 & N.S. \\
\hline Neutrophils & $19 / 33(57.6)$ & $23 / 27(85.2)$ & $21 / 21(100)$ & 0.0003 & N.S. \\
\hline Mononuclear cells & $6 / 33(18.2)$ & $11 / 27(40.7)$ & $18 / 21(85.7)$ & $<0.0001$ & 0.0026 \\
\hline
\end{tabular}

Age, mean tumor diameter, and proliferative zone extension distance data are expressed as mean \pm SD (standard deviation)

Data are presented as numbers $(\%)$

CI confidence interval

higher in the $H$. pylori-uninfected group. While no significant difference was observed in the color of the tumors, there were many discolored lesions in both groups. The mean intercrypt distance ratios in the two groups were 1.96 (95\% confidence interval [CI] 1.70-2.21) and 1.59 (95\% CI 1.40-1.78), and the diagnostic accuracy rates were 100\% (21/21) and $69.7 \%$ (23/33). Both values were significantly higher in the H. pylori-uninfected group. No significant difference was observed between the groups in proliferative zone extension. The endoscopic findings of the APC-marked sites in the $H$. 
pylori-uninfected group showed extended intervening parts in 41 sites and wavy microvessels in 1 site. In the non- $H$. pylori eradication group, there were 42 sites with extended intervening parts, 17 sites with wavy microvessels, and 7 sites with a corkscrew pattern. The $H$. pylori-uninfected group included significantly more sites with extended intervening parts. Regarding atrophy around the lesion, although no atrophy was observed in the $H$. pylori-uninfected group, 21 lesions in the non- $H$. pylori eradication group were adjacent to an atrophic area. Atrophy was significantly more common in the non- $H$. pylori eradication group. Using the updated Sydney classification for comparison, the state of the background mucosa was evaluated to be significantly milder in the $H$. pylori-uninfected group for all items.

The H. pylori-uninfected group was also compared with the $H$. pylori eradication group. In comparison of the H. pyloriuninfected group to the $H$. pylori eradication group, there were more macroscopically flat-type lesions, the tumor diameter was significantly smaller, and the proportions of intramucosal and signet ring cell carcinomas were significantly higher in the $H$. pylori-uninfected group. While no significant difference was observed in tumor color, many lesions were discolored in both groups. In the following section, the data for the H. pylori-uninfected group are presented first, followed by those for the $H$. pylori eradication group. The mean intercrypt distance ratios were 1.96 (95\% CI 1.70-2.21) in the $H$. pylori-uninfected group and 1.94 (95\% CI 1.69-2.20) in the H. pylori eradication group, and the diagnostic accuracy rates were $100 \%$ (21/21) and 92.6\% (25/27), respectively. No significant differences were observed in either value, or in proliferative zone extension. The endoscopic findings of the APCmarked sites in the $H$. pylori-uninfected group were extended to intervening parts in 41 sites and wavy microvessels in 1 site. In the $H$. pylori eradication group, there were 38 sites with extended intervening parts, 12 sites with wavy microvessels, and 4 sites with a corkscrew pattern. The $H$. pylori-uninfected group included significantly more sites with extended intervening parts. Regarding atrophy around the lesion, 12 lesions in the $H$. pylori eradication group were adjacent to an atrophic area. Atrophy was significantly more common in the $H$. pylori eradication group.

Comparison of background mucosa (updated Sydney classification) showed that atrophy and monocyte infiltration were significantly milder in the $H$. pylori-uninfected group. However, no significant difference was observed in the severity of neutrophil infiltration and intestinal metaplasia.

\section{Discussion}

In patients undergoing ESD for UD-type EGCs, the surgical margin-positive rate and the curative resection rate vary greatly among institutions, ranging from 1.7 to $52.6 \%$ and from 63.9 to $82.5 \%$, respectively [15-24]. In our previous study, diagnostic demarcation accuracy was obtained with ME-NBI in approximately $80 \%$ of patients undergoing ESD for UD-type EGCs [5].

Thus, to determine whether the rate of diagnostic accuracy was actually higher in the $H$. pylori-uninfected group than in the non- $H$. pylori eradication group and whether the rate after resolution of inflammatory cell infiltration achieved by $H$. pylori eradication was different from the rate in the $H$. pylori-uninfected group, in which inflammatory cell infiltration caused by $H$. pylori was absent from the beginning, we compared and analyzed the $H$. pylori-uninfected, non- $H$. pylori eradication, and $H$. pylori eradication groups in the present study.

Compared with the non- $H$. pylori eradication and $H$. pylori eradication groups, the $H$. pylori-uninfected group included more macroscopically flat-type lesions. As previously reported, we consider this finding to be attributable to the fact that in the $H$. pylori-uninfected group, tumors were often confined to the proliferative zone in the early stages [11], and cancer was neither exposed nor formed a depression on the surface. Many intramucosal carcinomas were observed. In addition, as we have previously reported, intramucosal lesions are common in $H$. pylori-uninfected patients because tumor cells have a low proliferative capacity and remain in the proliferative zone of the mucosa in many cases.

Furthermore, it has been reported that signet ring cell carcinoma is common in $H$. pylori-uninfected patients [10, $12]$. We consider the results of the present study to be consistent with this finding.

The smaller tumor diameter observed in the H. pyloriuninfected group may be attributable to the low proliferative capacity of the tumor cells, as previously stated [11]. We have also previously reported that inflammatory cell infiltration renders diagnosis of demarcation difficult [7], meaning that the tumors are difficult to identify. In other words, inflammatory cell infiltration makes the detection of tumors difficult. Conversely, without inflammatory cell infiltration, tumors may be easily detectable. These factors suggest that, in the $H$. pylori-uninfected group, cancer might have been detected at a small size. Thus, the tumor diameter in the $H$. pylori-uninfected group was presumably smaller than that in the $H$. pylori eradication group.

The mean intercrypt distance ratio and the rate of diagnostic accuracy in the $H$. pylori-uninfected group were significantly higher than the corresponding values in the non$H$. pylori eradication group, but no significant differences were observed in these parameters between the $H$. pyloriuninfected and $H$. pylori eradication groups. Moreover, the state of the background mucosa was milder in the $H$. pyloriuninfected group than in the non- $H$. pylori eradication group for all items included in the updated Sydney classification. 
Comparison between the $H$. pylori-uninfected and $H$. pylori eradication groups showed that atrophy and monocyte infiltration were significantly milder in the $H$. pylori-uninfected group; however, no significant differences were observed in the severity of neutrophil infiltration and intestinal metaplasia. In our previous study comparing $H$. pylori eradication and non-H. pylori eradication groups, we reported that the diagnostic accuracy of demarcation is improved by the following mechanism: H. pylori eradication leads to resolution of neutrophil infiltration, which in turn results in reduction in extended intervening parts caused by inflammatory cell infiltration in the noncancerous region; consequently, the contrast between the cancerous and noncancerous regions becomes clear, and extended intervening parts in the cancerous region become apparent [7]. In the present study, when the $H$. pylori-uninfected and non- $H$. pylori eradication groups were compared, the rate of diagnostic accuracy was higher in the H. pylori-uninfected group because of the absence of inflammatory cell infiltration caused by $H$. pylori. However, comparison between the H. pylori-uninfected and $H$. pylori eradication groups revealed no difference in the severity of neutrophil infiltration, suggesting that $H$. pylori eradication might have reduced the severity of neutrophil infiltration in the H. pylori eradication group to a level comparable to that of the H. pylori-uninfected group. For this reason, the rate of diagnostic accuracy of demarcation might also have been improved in the $H$. pylori eradication group, and no significant difference was observed in the diagnostic accuracy.

Regarding intestinal metaplasia in the H. pylori eradication group, since the infection was eradicated prior to progression of the lesion to intestinal metaplasia, there was considered to be no significant difference in this group as compared with the uninfected group.

The diagnosis of demarcation is difficult following $H$. pylori eradication because the surface of the cancerous region is covered with gastritis-like tissue (mucosal tissue resembling gastritis around the lesion) $[25,26]$. H. pylori eradication has also been reported to flatten lesions of differentiated-type early gastric cancers [27, 28]. However, the majority of UD-type EGCs are macroscopically classified as the superficial flattened or depressed type, and many cases show no tumor exposure from the superficial layer regardless of whether H. pylori eradication has been performed, as the results of the present study also show. Therefore, the diagnostic accuracy of demarcation of UD-type EGCs is improved by resolution of neutrophil infiltration.

Although proliferative zone extension is reported to be a factor affecting the diagnosis of demarcation of undifferentiated-type cancer, in addition to extended intervening parts $[4,29]$, the present study did not show any significant difference either between the $H$. pylori-uninfected and non- $H$. pylori eradication groups or between the $H$. pylori-uninfected and $H$. pylori eradication groups. In previous reports, because the diagnostic ability of conventional routine endoscopy was not based on magnifying endoscopic observation, proliferative zone extension was an important cause of misdiagnosis by demarcation. However, it is assumed that the present study did not show any significant difference in proliferative zone extension because ME-NBI allowed detection of extended intervening parts.

Severe atrophy has also been reported to affect the diagnosis of demarcation [30, 31]. This finding seems to be attributable to the fact that in the presence of atrophy, the mucosal discoloration of undifferentiated-type cancer becomes unclear. In the present study, although atrophy was observed around 21 of the 33 lesions in the non- $H$. pylori eradication group and 12 of the 27 lesions in the H. pylori eradication group, it was not observed around any lesions in the $H$. pylori-uninfected group. In comparison of background mucosa, atrophy was also detected significantly less frequently in the $H$. pylori-uninfected group. Moreover, there were many discolored lesions in all groups. Although the non- $H$. pylori eradication and $H$. pylori eradication groups included significantly more lesions adjacent to an atrophic area, the accuracy rate of diagnostic demarcation was not significantly different from that of the $H$. pylori-uninfected group in either the non-eradication or eradication group. The results of the present study suggested that atrophy does not make diagnosis of demarcation more difficult.

The most common finding of the APC-marked sites was an extended intervening part in the $H$. pylori-uninfected, non-H. pylori eradication, and H. pylori eradication groups. If inflammation is resolved, intervening parts extended by cancer can easily be recognized with ME-NBI. Thus, it was suggested that borders with atrophic areas might be visible even for lesions located closely to an atrophic area.

\section{Limitations}

One of the limitations of this study is its retrospective design. In addition, it is not a comparative study. Outcomes in actual clinical practice may differ from the results of this study, depending on the endoscopist. Although the utmost oral and utmost anal sites of the lesion were marked with APC, there may be other sites that make diagnosis of demarcation difficult or easy. When ESD is performed in our hospital, the oral and anal aspects of the area to be resected are usually marked to distinguish which is the oral and which is the anal side of the resected specimen. In this study, we marked the most proximal oral and distal anal sides of the lesion. Another limitation is that this study was conducted at a single institution.

Differences in the diagnostic accuracy of demarcation among operators are difficult to assess in this study 
because our results were not compared with outcomes at other institutions. Although this study was conducted at a single institution, we assume that because cases had accumulated at a cancer center over 5 years, the study included a sufficient number of patients with relatively uncommon UD-type EGCs who had and had not undergone H. pylori eradication. Lesions were demarcated by marking the utmost oral and utmost anal aspects, which proves that neither easy nor difficult parts in the lesions were deliberately selected for diagnosis of demarcation. This study thus appears to be adequately valuable.

In conclusion, the rate of diagnostic accuracy of demarcation was high in the $H$. pylori-uninfected group. Moreover, this study suggested that the accuracy rate in the H. pylori eradication group was comparable to that in the H. pyloriuninfected group.

\section{Compliance with ethical standards}

Conflict of interest There is no conflict of interest in this study.

Human rights statement and informed consent This study was conducted in compliance with the principles of the "Declaration of Helsinki" issued in 1964 and revised thereafter. Prior to registration, all personal identifiers were deleted and informed consent was obtained from all patients to allow the use of pathological specimens and imaging data for this study.

\section{References}

1. Japanese Gastric Cancer Association. Japanese gastric cancer treatment guidelines 2014 (ver. 4). Gastric Cancer. 2017;20:1-19.

2. Gotoda T, Yanagisawa A, Sasako M, Ono H, Nakanishi Y, Shimoda $\mathrm{T}$, et al. Incidence of lymph node metastasis from early gastric cancer: estimation with a large number of cases at two large centers. Gastric Cancer. 2000;4:219-25.

3. Hirasawa T, Gotoda T, Miyata S, Kato Y, Shimoda T, Taniguchi $\mathrm{H}$, et al. Incidence of lymph node metastasis and the feasibility of endoscopic resection for undifferentiated-type early gastric cancer. Gastric Cancer. 2009;12:148-52.

4. Ninomiya Y, Yanagisawa A, Kato Y, Tomimatsu H. Unrecognizable intramucosal spread of diffuse-type mucosal gastric carcinomas of less than $20 \mathrm{~mm}$ in size. Endoscopy. 2000;8:604-8.

5. Horiuchi Y, Fujisaki J, Yamamoto N, Shimizu T, Miyamoto Y, Tomida H, et al. Accuracy of diagnostic demarcation of undifferentiated-type early gastric cancers for magnifying endoscopy with narrow-band imaging: endoscopic submucosal dissection cases. Gastric Cancer. 2016;19:515-23.

6. Horiuchi Y, Fujisaki J, Yamamoto N, Shimizu T, Omae M, Ishiyama A, et al. Accuracy of demarcation of undifferentiated-type early gastric cancer for magnifying endoscopy with narrow band imaging: surgical cases. Surg Endosc. 2017;31:1906-13.

7. Horiuchi Y, Fujisaki J, Yamamoto N, Omae M, Ishiyama A, Yoshio T, et al. Diagnostic accuracy of demarcation of undifferentiated-type early gastric cancer after Helicobacter Pylori eradication. J Gastroenterol. 2017;52:1023-30.

8. Uemura N, Okamoto S, Yamamoto S, Matsumura N, Yamaguchi $\mathrm{S}$, Yamakido M, et al. Helicobacter pylori infection and the development of gastric cancer. N Engl J Med. 2001;345:784-9.
9. Correa P. Helicobacter pylori and gastric carcinogenesis. Am J Surg Pathol. 1995;19:S37-43.

10. Matsuo T, Ito M, Takata S, Yoshihara M, Chayama K. Low prevalence of Helicobacter pylori-negative gastric cancer among Japanese. Helicobacter. 2011;16:415-9.

11. Horiuchi Y, Fujisaki J, Yamamoto N, Shimizu T, Miyamoto $\mathrm{Y}$, Tomida $\mathrm{H}$, et al. Biological behavior of the intramucosal Helicobacter pylori-negative undifferentiated-type early gastric cancer: comparison with Helicobacter pylori-positive early gastric cancer. Gastric Cancer. 2016;19:160-5.

12. Horiuchi Y, Fujisaki J, Ishizuka N, Omae M, Ishiyama A, Yoshio T, et al. Study on clinical factors involved in Helicobacter pylori-uninfected, undifferentiated-type early gastric cancer. Digestion. 2017;96(4):213-9.

13. Yagi K, Nakamura A, Sekine A. Characteristic endoscopic and magnified endoscopic findings in the normal stomach without Helicobacter pylori infection. J Gastroenterol Hepatol. 2002;17:39-45.

14. Dixon MF, Genta RM, Yardley JH, Correa P. Classification and grading of gastritis: the updated Sydney System. International workshop on the histopathology of gastritis, Houston 1994. Am J Surg Pathol. 1996;20:1161-81.

15. Okada K, Fujisaki J, Kasuga A, Omae M, Hirasawa T, Ishiyama A, et al. Diagnosis of undifferentiated-typer early gastric cancers by magnification endoscopy with narrow-band imaging. J Gastroenterol Hepatol. 2011;26:1262-9.

16. Nakayoshi T, Tajiri H, Matsuda K, Kaise M, Ikegami M, Sasaki $\mathrm{H}$, et al. Magnifying endoscopy combined with narrow band imaging system for early gastric cancer: correlation of vascular pattern with histopathology (including video). Endoscopy. 2004;12:1080-4.

17. Kamada K, Tomatsuri N, Yoshida N. Endoscopic submucosal dissection for undifferentiated early gastric cancer as the expanded indication lesion. Digestion. 2012;85:111-5.

18. Okada K, Fujisaki J, Yoshida T, Ishikawa H, Suganuma T, Kasuga A, et al. Long-term outcomes of endoscopic submucosal dissection for undifferentiated-type early gastric cancer. Endoscopy. 2012;44:122-7.

19. Abe S, Oda I, Suzuki H, Nonaka S, Yoshinaga S, Odagaki T, et al. Short- and long-term outcomes of endoscopic submucosal dissection for undifferentiated early gastric cancer. Endoscopy. 2013;45:703-7.

20. Yamamoto Y, Fujisaki J, Hirasawa T, Ishiyama A, Yoshimoto $\mathrm{K}$, Ueki N, et al. Therapeutic outcomes of endoscopic submucosal dissection of undifferentiated-type intramucosal gastric cancer without ulceration and preoperatively diagnosed as 20 millimetres or less in diameter. Dig Endosc. 2010;22:112-8.

21. Kim YY, Jeon SW, Kim J, Park JC, Cho KB, Park KS, et al. Endoscopic submucosal dissection for early gastric cancer with undifferentiated histology: could we extend the criteria beyond? Surg Endosc. 2013;12:4656-62.

22. Kang HY, Kim SG, Kim JS, Jung HC, Song IS. Clinical outcomes of endoscopic submucosal dissection for undifferentiated early gastric cancer. Surg Endosc. 2010;24:509-16.

23. Kim JH, Lee YC, Kim H, Song KH, Lee SK, Cheon JH, et al. Endoscopic resection for undifferentiated early gastric cancer. Gastrointest Endosc. 2009;69:e1-9.

24. Park YD, Chung YJ, Chung HY, Yu W, Bae HI, Jeon SW, et al. Factors related to lymph node metastasis and the feasibility of endoscopic mucosal resection for treating poorly differentiated adenocarcinoma of the stomach. Endoscopy. 2008;40:7-10.

25. Ito $\mathbf{M}$, Tanaka $\mathrm{S}$, Takata $\mathrm{S}$, Oka $\mathrm{S}$, Imagawa $\mathrm{S}$, Ueda $\mathrm{H}$, et al. Morphological changes in human gastric tumours after eradication therapy of Helicobacter pylori in a short-term follow-up. Aliment Pharmacol Ther. 2005;21:559-66. 
26. Kobayashi M, Hashimoto S, Nishikura K, Mizuno K, Takeuchi M, Sato Y, et al. Magnifying narrow-band imaging of surface maturation in early differentiated-type gastric cancers after Helicobacter pylori eradication. J Gastroenterol. 2013;48:1332-42.

27. Yamamoto K, Kato M, Takahashi M, et al. Clinicopathological analysis of early-stage gastric cancers detected after successful eradication of Helicobacter pylori. Helicobacter. 2011;16:210-6.

28. Matsuo T, Ito M, Tatsugami M, et al. Gastric cancer development after Helicobacter pylori eradication therapy: a new form of gastric neoplasia. Digestion. 2012;85:61-7.

29. Sawada S, Fujisaki J, Yamamoto N, Kato Y, Ishiyama A, Ueki $\mathrm{N}$, et al. Expansion of indications for endoscopic treatment of undifferentiated mucosal gastric cancer: analysis of intramucosal spread in resected specimens. Dig Dis Sci. 2010;55(5):1376-80.

30. Kim JH, Kim YH, Jung DH, Jeon HH, Lee YC, Lee H, et al. Follow-up outcomes of endoscopic resection for early gastric cancer with undifferentiated histology. Surg Endosc. 2014;28(9):2627-33.

31. Kiyotoki S, Nishikawa J, Satake M, Fukagawa Y, Shirai Y, Hamabe K, et al. Usefulness of magnifying endoscopy with narrow-band imaging for determining gastric tumor margin. $\mathrm{J}$ Gastroenterol Hepatol. 2010;25(10):1636-41. 